\title{
Unified quantum invariants and their refinements for homology 3-spheres with 2-torsion
}

\author{
by \\ Anna Beliakova (Zürich), Christian Blanchet (Paris) and \\ Thang T. Q. Lê (Atlanta, GA)
}

\begin{abstract}
For every rational homology 3 -sphere with $H_{1}(M, \mathbb{Z})=(\mathbb{Z} / 2 \mathbb{Z})^{n}$ we construct a unified invariant (which takes values in a certain cyclotomic completion of a polynomial ring) such that the evaluation of this invariant at any odd root of unity provides the $\mathrm{SO}(3)$ Witten-Reshetikhin-Turaev invariant at this root, and at any even root of unity the SU(2) quantum invariant. Moreover, this unified invariant splits into a sum of the refined unified invariants dominating spin and cohomological refinements of quantum SU(2) invariants. New results on the Ohtsuki series and the integrality of quantum invariants are the main applications of our construction.
\end{abstract}

Introduction. The Witten-Reshetikhin-Turaev (WRT) invariants of 3-manifolds, also known as quantum invariants, are defined only when the quantum parameter $q$ is a certain root of unity. In [5], Habiro proposed a construction of a unified invariant of integral homology 3 -spheres, dominating all quantum $\mathrm{SU}(2)$ invariants.

The unified invariant is an element of the Habiro ring

$$
\widehat{\mathbb{Z}[q]}:=\varliminf_{n} \frac{\mathbb{Z}[q]}{(1-q)\left(1-q^{2}\right) \ldots\left(1-q^{n}\right)} .
$$

Every element $f \in \widehat{\mathbb{Z}[q]}$ can be written as an infinite sum

$$
f(q)=\sum_{k \geq 0} f_{k}(q)(1-q)\left(1-q^{2}\right) \ldots\left(1-q^{k}\right),
$$

with $f_{k}(q) \in \mathbb{Z}[q]$. If $\xi$ is a root of unity, then $f(\xi)$ is well-defined, since the summands become zero if $k$ is bigger than the order of $\xi$. The Habiro ring has remarkable properties and is very suitable for the study of quantum invariants. The result of Habiro mentioned above is

2000 Mathematics Subject Classification: Primary 57N10; Secondary 57M25.

Key words and phrases: quantum invariants, Jones polynomial, Ohtsuki series, cyclotomic completion ring, $q$-hypergeometric series. 
Theorem 1 (Habiro). For every integral homology 3-sphere $M$, there exists an invariant $I_{M}(q) \in \widehat{\mathbb{Z}[q]}$ such that if $\xi$ is a root of unity, then $I_{M}(\xi)$ is the WRT invariant at $\xi$.

Let us mention the most important consequences of Habiro's construction. First of all, each product

$$
(q ; q)_{n}:=(1-q)\left(1-q^{2}\right) \ldots\left(1-q^{n}\right)
$$

is divisible by $(1-q)^{n}$, hence it is easy to expand every $f(q) \in \widehat{\mathbb{Z}[q]}$ into formal power series in $q-1$, denoted by $T(f)$ and called the Taylor series of $f(q)$ at $q=1$. One important property of $\widehat{\mathbb{Z}[q]}$ is that $f \in \widehat{\mathbb{Z}[q]}$ is uniquely determined by its Taylor series. In other words, the map $T: \widehat{\mathbb{Z}[q]} \rightarrow \mathbb{Z}[[q-1]]$ is injective. In particular, $\widehat{\mathbb{Z}[q]}$ is an integral domain. Moreover, every $f \in$ $\widehat{\mathbb{Z}[q]}$ is determined by the values of $f$ at any infinite set of roots of unity of prime power order. From the existence of $I_{M}$ one can derive the following consequences for integral homology spheres:

- The quantum invariants at all roots of unity are algebraic integers.

- The quantum invariants at any infinite set of roots of unity of prime power order determine the whole set of quantum invariants.

- The Ohtsuki series (see $[18,12])$ has integer coefficients and determines the whole set of quantum invariants.

- The Le-Murakami-Ohtsuki invariant (see [13]) totally determines the quantum invariants.

The integrality of quantum invariants was established earlier only at roots of unity of prime order (see $[15,11]$ ). The integrality of the Ohtsuki series was proved by Rozansky, using quite a different method.

In [10], the third author extended Habiro's results to rational homology 3-spheres. More precisely, for a 3-manifold $M$ with $\left|H_{1}(M, \mathbb{Z})\right|=a$, he constructed a unified invariant $I_{M}$ dominating quantum $\mathrm{SO}(3)$ invariants of $M$ at roots of unity of order odd and coprime to $a$. The Habiro ring was modified by inverting $a$ and all cyclotomic polynomials not coprime to $a$.

More precisely, for $t:=q^{1 / a}$, let $\mathcal{R}_{a, k}$ be the subring of $\mathbb{Q}(t)$ generated over $\mathbb{Z}\left[t^{ \pm 1}\right]$ by $(t ; t)_{k} /(q ; q)_{k}$. Note that

$$
\mathcal{R}_{a, 1} \subset \mathcal{R}_{a, 2} \subset \cdots \subset \mathcal{R}_{a},
$$

where $\mathcal{R}_{a}=\bigcup_{k=1}^{\infty} \mathcal{R}_{a, k}$. Let

$$
\widehat{\mathcal{R}}_{a}:=\lim _{n} \frac{\mathcal{R}_{a}}{(q ; q)_{n}}
$$

be its cyclotomic completion. Every element $f \in \widehat{\mathcal{R}}_{a}$ has the following presentation: 


$$
f=\sum_{n=0}^{\infty} f_{n}(t) \frac{\left(1-q^{n+1}\right)\left(1-q^{n+2}\right) \ldots\left(1-q^{2 n+1}\right)}{1-q},
$$

where $f_{n}(t) \in \mathcal{R}_{a}$. It was shown in [10] that $I_{M} \in \widehat{\mathcal{R}}_{a}$.

Let $\Gamma_{a}$ be the set of all elements of $\widehat{\mathcal{R}}_{a}$ that have presentation (1) such that $f_{n}(t) \in \mathcal{R}_{a, 2 n+1}$. In [2], the first and third authors proved that $\Gamma_{a}$ is smaller than $\widehat{\mathcal{R}}_{a}$ and that $I_{M} \in \Gamma_{a}$, i.e. the unified invariant has even stronger integrality.

The results in [10] and [2] concern only the $\mathrm{SO}(3)$ invariant, for which the order of quantum parameter must be odd. In this paper we mainly address the case of $\mathrm{SU}(2)$ when the order is even. We construct a unified invariant dominating quantum $\mathrm{SO}(3)$ and $\mathrm{SU}(2)$ invariants of rational homology 3 -spheres with 2-torsion only. We show that our unified invariant splits into a sum of refined unified invariants dominating spin and cohomological refinements of quantum $\mathrm{SU}(2)$ invariants. New results on integrality of quantum invariants and the Ohtsuki series are the main applications of our construction.

0.1. Results. Let $\mathcal{M}_{n}$ be the set of all oriented closed compact 3-manifolds $M$ with $H_{1}(M, \mathbb{Z})=(\mathbb{Z} / 2 \mathbb{Z})^{n}$. When $M$ is not an integral homology 3 -sphere, the WRT SU(2) invariant, denoted here by $\tau_{M}(\xi)$, depends on a 4 th root of $\xi$, although we will not indicate this in our notation.

Let $M \in \mathcal{M}_{n}$. If $\xi$ is an odd root of unity, then $\tau_{M}(\xi)=0$, but $\tau_{M}^{\mathrm{SO}(3)}(\xi)$ $\neq 0$. In this case, we choose $\zeta$ to be the square root of $\xi$ which has the same order as $\xi$, and put

$$
\tau_{M}^{\prime}(\xi)=\tau_{M}^{\mathrm{SO}(3)}(\xi) /\left(\tau_{\mathbb{R} P^{3}}^{\mathrm{SO}(3)}(\xi)\right)^{n},
$$

where $\mathbb{R} P^{3}$ is the projective space.

If the order of $\xi$ is even (then the order of $\zeta$ is divisible by 4 ), define

$$
\tau_{M}^{\prime}(\xi)=\tau_{M}(\xi) /\left(\tau_{\mathbb{R} P^{3}}(\xi)\right)^{n} .
$$

Note that the quantum invariant $\tau_{M}^{\prime}(\xi)$ depends only on a square root of $\xi$.

For $q=v^{2}$, we define $\Gamma:=\mathbb{Z}[1 / 2][v]$. Let $S=\{n \in \mathbb{N} \mid n \not \equiv 2(\bmod 4)\}$, where $\mathbb{N}=\{1,2, \ldots\}$. The cyclotomic completion $\Gamma^{S}$ of the polynomial ring $\Gamma$ with respect to $S$ was defined by Habiro in [6] (the definition is recalled in Section 2).

Suppose $\xi$ is a root of unity. Fix a square root $\zeta$ of $\xi$ such that if the order of $\xi$ is odd, then the order of $\zeta$ is the same as that of $\xi$. Then one can evaluate every element $f \in \Gamma^{S}$ by replacing $v$ with $\zeta$; the result is a complex number, denoted by $\operatorname{ev}_{\xi}(f)$.

Our first main result is 
THeOREM 2. For every $M \in \mathcal{M}_{n}$, there exists a unique invariant $I_{M} \in \Gamma^{S}$ such that for every root $\xi$ of unity and a choice of its square root $\zeta$ as above, one has $\operatorname{ev}_{\xi}\left(I_{M}\right)=\tau_{M}^{\prime}(\xi)$.

Hence, $I_{M}$ unifies $\mathrm{SO}(3)$ and $\mathrm{SU}(2)$ invariants of $M \in \mathcal{M}_{n}$ at all roots of unity. Moreover, in Section 5 we give an explicit formula for this invariant.

Corollary 3. For $M \in \mathcal{M}_{n}$, and the quantum invariants $\tau_{M}^{\prime}$, the following statements hold.

(a) The quantum invariants belong to $\mathbb{Z}[1 / 2][\zeta]$.

(b) The quantum invariants are determined by their values at roots of unity $\zeta$ with $\operatorname{ord}(\zeta) \in\left\{2^{k} p^{e} \mid k \in \mathbb{N}, e \in \mathbb{N}\right\}$ for any odd prime $p$.

Clearly, part (b) also holds for $\tau_{M}$, but for (a) to be true, one may need to multiply $\tau_{M}$ by $\zeta^{1 / 2}$.

OpEn PROBLEM. We do not know whether these invariants are determined by the Le-Murakami-Ohtsuki invariant or not.

Spin and cohomological refinements. Suppose now the order of $\xi$ is even, i.e. the order of $\zeta$ is divisible by 4 . There are refined quantum invariants $\tau_{M, \sigma}(\xi)$, defined in [1], [9], where $\sigma$ is a spin structure or a cohomological class in $H^{1}(M, \mathbb{Z} / 2 \mathbb{Z})$, depending on whether the order of $\zeta$ is congruent to $0(\bmod 8)$ or $4(\bmod 8)$.

We will renormalize $\tau_{M, \sigma}$ by dividing by the non-refined invariant of the projective space, i.e. $\tau_{M, \sigma}^{\prime}(\xi):=\tau_{M, \sigma}(\xi) /\left(\tau_{\mathbb{R} P^{3}}(\xi)\right)^{n}$. Then we have $\tau_{M}^{\prime}(\xi)=$ $\sum_{\sigma} \tau_{M, \sigma}^{\prime}(\xi)$.

For $T=\{n \in \mathbb{N} \mid n \equiv 0(\bmod 8)\}$, let $\Gamma^{T}$ be the cyclotomic completion with respect to $T$ as defined in Section 2. If $\zeta$ is a root of unity with order in $T$, then we can evaluate any element $f \in \Gamma^{T}$ by replacing $v$ with $\zeta$; the result is a complex number denoted by $\operatorname{ev}_{\xi}(f)$.

Our next result is

Theorem 4. For any $M \in \mathcal{M}_{n}$ and a spin structure $\sigma$ on $M$, there exists a unique spin invariant $I_{M, \sigma}(v) \in \Gamma^{T}$, dominating spin refinements of the quantum invariants $\tau_{M, \sigma}^{\prime}(\xi)$ at all roots of unity $\xi$ whose order is divisible by 4.

A similar statement concerning cohomological refinements is given in Theorem 8, where the unified invariant is an element of $\Gamma^{S_{2}}$ with $S_{2}=$ $\{4(2 n+1) \mid n \in \mathbb{N}\}$.

Corollary 5. Let $M \in \mathcal{M}_{n}$.

(a) The set of spin invariants $\left\{\tau_{M, \sigma}(\xi) \mid \operatorname{ord}(\xi) \equiv 0(\bmod 4)\right\}$ is determined by their values at roots of unity $\xi$ with $\operatorname{ord}(\xi) \in\left\{2^{k} p^{e} \mid k \geq 2\right.$, $e \in \mathbb{N}\}$ where $p$ is an odd prime. 
(b) The set of cohomological refinements $\left\{\tau_{M, \sigma}(\xi) \mid \operatorname{ord}(\xi) \equiv 2 \bmod 4\right\}$ is determined by their values at roots of unity $\xi$ with $\operatorname{ord}(\xi) \in\left\{2 p^{e} \mid\right.$ $e \in \mathbb{N}\}$ where $p$ is an odd prime.

0.2. The case $\left|H_{1}\right|=2$. Suppose $M \in \mathcal{M}_{1}$. Let us consider the ring

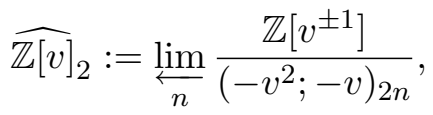

where

$$
\begin{aligned}
\left(-v^{2} ;-v\right)_{2 n} & :=\prod_{i=2}^{2 n+1}\left(1+(-v)^{i}\right) \\
& =\left(1-v^{3}\right)\left(1-v^{5}\right) \ldots\left(1-v^{2 n+1}\right)(1+q)\left(1+q^{2}\right) \ldots\left(1+q^{n}\right) .
\end{aligned}
$$

Every $f(v) \in \widehat{\mathbb{Z}[v]_{2}}$ can be written as, with $f_{n}(v) \in \mathbb{Z}\left[v^{ \pm 1}\right]$,

$$
f(v)=\sum_{n=0}^{\infty} f_{n}(v)\left(-v^{2} ;-v\right)_{2 n}
$$

If $\zeta$ is a root of unity of order either odd or divisible by 4 , then the evaluation $\operatorname{ev}_{\xi}(f(v))=\left.f(v)\right|_{v=\zeta}$ is well-defined. For every root $\xi$ of unity, one can choose a square root $\zeta$ of $\xi$ whose order is either odd or divisible by 4 .

Let

$$
\widehat{\mathbb{Z}[v]_{s}}:={\underset{\lim }{n}}_{n} \frac{\mathbb{Z}[v]}{(1+q)\left(1+q^{2}\right) \ldots\left(1+q^{n}\right)} .
$$

If $\zeta$ is a root of unity of order divisible by 4 , then $f(\zeta)$ is well-defined for $f(v) \in \widehat{\mathbb{Z}[v]_{s}}$.

TheOREM 6. For every $M \in \mathcal{M}_{1}$, there exists a unique invariant $I_{M}(v)$ $\in \widehat{\mathbb{Z}[v]_{2}}$ such that $\operatorname{ev}_{\xi}\left(I_{M}(v)\right)=\tau_{M}^{\prime}(\xi)$ for any root of unity $\xi$. Moreover, $I_{M}(v)=\sum_{\sigma} I_{M, \sigma}(v)$ where $\sigma$ runs over spin structures on $M$, and $I_{M, \sigma}(v) \in$ $\frac{1}{1-v} \widehat{\mathbb{Z}[v]_{S}}$ dominates the refined quantum invariants $\tau_{M, \sigma}(\xi)$.

The ring $\widehat{\mathbb{Z}[v]_{2}}$ is smaller than the ring $\Gamma^{S}$ of Theorem 2 and also than the ring $\Gamma_{2}$ used in [2], because of the factors $(1+q)\left(1+q^{2}\right) \ldots\left(1+q^{n}\right)$ in the completion, which are responsible for the spin and cohomological refinements. Hence, when restricted to $\mathrm{SO}(3)$ invariants, the integrality stated in Theorem 6 is stronger than that in [2].

By results of $[6], \widehat{\mathbb{Z}[v]_{2}}$ embeds in $\mathbb{Z}[[v-1]]$, via Taylor series (cf. Proposition 2.1 below). As a consequence, we will prove

Corollary 7. For $M \in \mathcal{M}_{1}$ and the quantum invariants $\tau_{M}^{\prime}$, the following statements hold.

(a) The quantum invariants at all roots of unity are algebraic integers. 
(b) The quantum invariants at any infinite set of roots of unity of odd prime power order determine the whole set of quantum invariants.

(c) The Ohtsuki series of $M$, a formal power series in $q-1$, has coefficients in $\mathbb{Z}[1 / 2]$. If $\zeta$ is a root of unity of order $p^{d}$ with $p$ an odd prime, then the Ohtsuki series at $\zeta$ converges p-adically to the quantum invariant at $\zeta$.

(d) The Le-Murakami-Ohtsuki invariant determines the quantum invariants at all roots of unity.

The integrality of $\tau_{M, \sigma}$ for $\mathbb{Z} / p \mathbb{Z}$-homology spheres at roots of order $2 p$, where $p$ is an odd prime and $\sigma$ is a cohomological class, was studied by Murakami in [16, 17].

ExAmple. Suppose $M$ is obtained by surgery on the figure 8 knot with framing 2. Then

$$
I_{M}(v)=\sum_{n=0}^{\infty} v^{-n(n+2)}\left(-v^{2} ;-v\right)_{2 n} .
$$

(a) Spin refinement. Let $\sigma_{0}$ be the characteristic spin structure on $M$, and $\sigma_{1}$ the other one. Then

$I_{M, \sigma_{\varepsilon}}(v)$

$=\frac{1}{2(1-v)} \sum_{n=0}^{\infty} v^{-n(n+2)} \prod_{i=1}^{n}\left(1+q^{i}\right)\left[\prod_{i=0}^{n}\left(1-v^{2 i+1}\right)-(-1)^{\varepsilon+n} \prod_{i=0}^{n}\left(1+v^{2 i+1}\right)\right]$.

Suppose that $\operatorname{ord}(\zeta) \equiv 0(\bmod 8)$ and $\operatorname{ord}(\zeta) / 8 \equiv \chi(\bmod 2)$. Then $\operatorname{ev}_{\xi}\left(I_{M, \sigma_{\varepsilon}}(v)\right)=\tau_{M, \sigma_{\varepsilon+\chi}}(\zeta)$.

(b) Cohomological refinement. Let $\sigma_{\varepsilon} \in H^{1}(M, \mathbb{Z} / 2 \mathbb{Z})$, and $\sigma_{1}$ be trivial. Then

$I_{M, \sigma_{\varepsilon}}(v)$

$=\frac{1}{2(1-v)} \sum_{n=0}^{\infty} v^{-n(n+2)} \prod_{i=1}^{n}\left(1+q^{i}\right)\left[\prod_{i=0}^{n}\left(1-v^{2 i+1}\right)+(-1)^{\varepsilon+n} I \prod_{i=0}^{n}\left(1+v^{2 i+1}\right)\right]$.

Assume that $\operatorname{ord}(\zeta)=4 k$ with odd $k$ and $\zeta^{k^{2}}=(-1)^{\chi} I$, where $I$ is the unit complex number. Then $\operatorname{ev}_{\xi}\left(I_{M, \sigma_{\varepsilon}}(v)\right)=\tau_{M, \sigma_{\varepsilon+\chi}}(\zeta)$.

0.3. Plan of the paper. The paper is organized as follows. After recalling the definitions, we collect the results on cyclotomic completions of polynomial rings in Section 2. Then we introduce the Laplace transform method for constructing unified invariants. Applying this method to integral homology 3-spheres, we get precise formulas for Habiro's unified invariants. Next, we apply this method to $M \in \mathcal{M}_{n}$. Here again the exact formula for the Laplace transform implies various above mentioned results. In Section 6, we construct spin and cohomological refinements of the unified invariant. 
Acknowledgments. The first author wishes to express her gratitude to Dennis Stanton for a significant simplification of the proofs of Lemmas 4.2 and 5.2.

1. The colored Jones polynomial and the WRT invariant. Let us first fix the notation. Throughout the paper, $q=v^{2}$ and

$$
\{n\}:=v^{n}-v^{-n}, \quad\{n\} !:=\prod_{i=1}^{n}\{i\}, \quad[n]:=\frac{\{n\}}{\{1\}}, \quad\left[\begin{array}{l}
n \\
k
\end{array}\right]:=\frac{\{n\} !}{\{k\} !\{n-k\} !} .
$$

Let $(a ; t)_{k}:=(1-a)(1-a t) \ldots\left(1-a t^{k-1}\right)$ and for simplicity $(q)_{n}:=(q ; q)_{n}$.

1.1. The colored Jones polynomial. Suppose $L$ is a framed, oriented link in $S^{3}$ with $m$ ordered components. For every positive integer $n$ there is a unique irreducible $\mathrm{sl}_{2}$-module $V_{n}$ of dimension $n$. For positive integers $n_{1}, \ldots, n_{m}$ one can define the quantum invariant $J_{L}\left(n_{1}, \ldots, n_{m}\right):=$ $J_{L}\left(V_{n_{1}}, \ldots, V_{n_{m}}\right)$ known as the colored Jones polynomial of $L$ (see e.g. [19]). Let us recall here a few well-known formulas. For the unknot $U$ with 0 framing one has

$$
J_{U}(n)=[n]=\{n\} /\{1\} .
$$

If $L^{\prime}$ is obtained from $L$ by increasing the framing of the $i$ th component by 1 , then

$$
J_{L^{\prime}}\left(n_{1}, \ldots, n_{m}\right)=q^{\left(n_{i}^{2}-1\right) / 4} J_{L}\left(n_{1}, \ldots, n_{m}\right) .
$$

In general, $J_{L}\left(n_{1}, \ldots, n_{m}\right) \in \mathbb{Z}\left[q^{ \pm 1 / 4}\right]$. However, there is a number $a \in$ $\{0,1 / 4,1 / 2,3 / 4\}$ such that $J_{L}\left(n_{1}, \ldots, n_{m}\right) \in q^{a} \mathbb{Z}\left[q^{ \pm 1}\right]$.

1.2. Evaluation map and Gauss sum. Throughout this paper, let $\xi$ be a primitive root of unity of order $r$. We first define, for each $\xi$, the evaluation map ev $\xi$, which replaces $q$ by $\xi$. Suppose that $r$ is odd. Then there exists an integer $4^{*}$, unique modulo $r$, such that $\left(\xi^{4^{*}}\right)^{4}=\xi$. We define $\operatorname{ev}_{\xi}\left(q^{1 / 4}\right):=\xi^{4^{*}}$.

If $r$ is even, then $\operatorname{ev}_{\xi}$ depends on a square root $\zeta$ of $\xi$. For $f \in \mathbb{Q}\left[v^{ \pm 1}\right]$, we define

$$
\mathrm{ev}_{\xi} f:=\left.f\right|_{v=\zeta} .
$$

Suppose $f\left(v ; n_{1}, \ldots, n_{m}\right)$ is a function of variables $v$ and integers $n_{1}, \ldots, n_{m}$. Let

$$
\sum_{n_{i}}^{\xi} f:=\sum_{n_{i}} \operatorname{ev}_{\xi}(f)
$$

where the $n_{i}$ run each over the set of numbers between 0 and $2 r-1$. Moreover, we define

$$
\sum_{n_{i}}^{\xi, \varepsilon} f:=\sum_{n_{i} \equiv \varepsilon(\bmod 2)} \operatorname{ev}_{\xi}(f)
$$


where for $\varepsilon=0$ (resp. $\varepsilon=1$ ), the $n_{i}$ in the sum run each over the set of even (resp. odd) numbers between 0 and $2 r-1$.

Variations of the Gauss sum are defined by the following formulas. Fix a 4 th root of $\xi$. For $b \in \mathbb{Z}$ let

Furthermore,

$$
\gamma_{b}(\xi):=\sum_{n}^{\xi} q^{b\left(n^{2}-1\right) / 4}, \quad \gamma_{b}^{\varepsilon}(\xi):=\sum_{n}^{\xi, \varepsilon} q^{b\left(n^{2}-1\right) / 4} .
$$

$$
F_{L}(\xi):=\sum_{n_{i}}^{\xi} J_{L}\left(n_{1}, \ldots, n_{m}\right) \prod_{i=1}^{m}\left[n_{i}\right] .
$$

For any sequence $c=\left(c_{1}, \ldots, c_{m}\right) \in(\mathbb{Z} / 2 \mathbb{Z})^{m}$ we define

$$
F_{L}^{c}(\xi):=\sum_{n_{i}}^{\xi, c_{i}+1} J_{L}\left(n_{1}, \ldots, n_{m}\right) \prod_{i=1}^{m}\left[n_{i}\right] .
$$

For $\varepsilon=0$ or 1 , let $F_{L}^{\varepsilon}(\xi):=F_{L}^{(\varepsilon, \ldots, \varepsilon)}(\xi)$.

1.3. Quantum (WRT) invariants and their refinements. All 3-manifolds in this paper are supposed to be compact, closed and oriented. Every link in a 3-manifold is framed, oriented, and has components ordered.

Suppose $M$ is an oriented 3-manifold obtained from $S^{3}$ by surgery along a framed, oriented link $L$. (Note that $M$ does not depend on the orientation of $L$.) Let $\sigma_{+}$(respectively, $\sigma_{-}$) be the number of positive (resp. negative) eigenvalues of the linking matrix of $L$. Suppose $\xi$ is a root of unity of order $r$ together with a fixed 4 th root of it. Then the WRT (or quantum) SU(2) invariant [19] is defined by

$$
\tau_{M}(\xi)=\frac{F_{L}(\xi)}{\left(F_{U^{+}}(\xi)\right)^{\sigma_{+}}\left(F_{U^{-}}(\xi)\right)^{\sigma_{-}}} .
$$

For connected sum, one has $\tau_{M \# N}(\xi)=\tau_{M}(\xi) \tau_{N}(\xi)$.

Suppose $\xi$ is a root of unity of odd order $r$. Then the quantum $\mathrm{SO}(3)$ invariant $[9]$ is defined by

$$
\tau_{M}^{\mathrm{SO}(3)}(\xi):=\frac{F_{L}^{1}(\xi)}{\left(F_{U^{+}}^{1}(\xi)\right)^{\sigma_{+}}\left(F_{U^{-}}^{1}(\xi)\right)^{\sigma_{-}}} .
$$

Let $L_{i j}$ be the $(i, j)$-entry of the linking matrix of $L$. Any solution $c=$ $\left(c_{1}, \ldots, c_{m}\right)$ of the characteristic equation $\sum_{j} L_{i j} c_{j}=L_{i i}(\bmod 2)$ defines a spin structure $\sigma_{c}$ on $M$ (see [9]). If the order of $\xi$ is divisible by 4 , then there exists an invariant of the pair $\left(M, \sigma_{c}\right)$ defined as follows:

$$
\tau_{M, \sigma_{c}}(\xi)=\frac{F_{L}^{c}(\xi)}{\left(F_{U^{+}}(\xi)\right)^{\sigma_{+}}\left(F_{U^{-}}(\xi)\right)^{\sigma_{-}}} .
$$

If the order of $\xi$ is $2(\bmod 4)$, then $(4)$ defines an invariant of the pair $\left(M, \sigma_{c}\right)$, where $\sigma_{c} \in H^{1}(M, \mathbb{Z} / 2 \mathbb{Z})$ is determined by the solution $c$ of the equation $\sum_{j} L_{i j} c_{j}=0(\bmod 2)$. In [9], [3] it is proved that $\tau_{M}(\xi)=$ $\sum_{\sigma} \tau_{M, \sigma}(\xi)$. 
1.4. Habiro's cyclotomic expansion of the colored Jones polynomial. For a link $L$ with $m$ components, define

$$
J_{L}^{\prime}\left(n_{1}, \ldots, n_{m}\right):=\frac{J_{L}\left(n_{1}, \ldots, n_{m}\right)}{\left[n_{1}\right] \ldots\left[n_{m}\right]} .
$$

Let $K$ be a knot with framing zero. Note that $J_{K}^{\prime}(\lambda) \in \mathbb{Z}\left[q^{ \pm 1}\right]$ for integer $\lambda \geq 1$. In [5], Habiro proved that there exist $C_{K, k}(q) \in \mathbb{Z}\left[q^{ \pm 1}\right]$ such that

$$
J_{K}^{\prime}(\lambda)=\sum_{k=0}^{\infty} C_{K, k}(q)\left(q^{1+\lambda}\right)_{k}\left(q^{1-\lambda}\right)_{k} .
$$

The sum in (5) is finite, because the summands with $k \geq \lambda$ are zero. This expansion is called the cyclotomic expansion of the colored Jones polynomial. The non-trivial part here is that $C_{K, k}$ 's are Laurent polynomials in $q$ with integer coefficients.

EXAMPLES. Let $3_{1}, \overline{3}_{1}$ and $4_{1}$ denote the right-handed trefoil, the lefthanded trefoil and the figure 8 knot. We have

$$
\begin{gathered}
J_{3_{1}}^{\prime}(\lambda)=\sum_{k=0}^{\infty} q^{-k(k+2)}\left(q^{1+\lambda}\right)_{k}\left(q^{1-\lambda}\right)_{k}, \quad J_{3_{1}}^{\prime}(\lambda)=\sum_{k=0}^{\infty} q^{k}\left(q^{1+\lambda}\right)_{k}\left(q^{1-\lambda}\right)_{k}, \\
J_{4_{1}}^{\prime}(\lambda)=\sum_{k=0}^{\infty}(-1)^{k} q^{-k(k+1) / 2}\left(q^{1+\lambda}\right)_{k}\left(q^{1-\lambda}\right)_{k} .
\end{gathered}
$$

Note. The coefficients $C_{K, k}$ are computed for all twist knots in [14].

More generally, we have the following.

Proposition 1.1 (Habiro). Let $L$ be an algebraically split link of $m$ components. There exist $C_{L, \mathbf{k}}(v) \in \mathbb{Z}\left[v^{ \pm 1}\right]$ with $\mathbf{k}=\left(k_{1}, \ldots, k_{m}\right)$ such that

$$
\begin{aligned}
& J_{L}^{\prime}\left(n_{1}, \ldots, n_{m}\right) \\
& =\sum_{k \geq 0}\left(\sum_{\max k_{i}=k} C_{L, \mathbf{k}}(v)(1-q)^{m} \prod_{i=1}^{m} \frac{\left(q^{1+n_{i}}\right)_{k_{i}}\left(q^{1-n_{i}}\right) k_{i}}{\left(q^{k_{i}+1}\right)_{k_{i}+1}}\right) \frac{\left(q^{k+1}\right)_{k+1}}{(1-q)} .
\end{aligned}
$$

EXAMPLE. Let $L$ be the 0 -framed Whitehead link. The following formula was obtained by Habiro in [7]:

$$
J_{L}^{\prime}(\lambda, \mu)=\sum_{k=0}^{\infty}(-1)^{k} v^{-k(k+1)}(1-q) \frac{\left(q^{1+\mu}\right)_{k}\left(q^{1-\mu}\right)_{k}}{\left(q^{k+1}\right)_{k+1}}\left(q^{1+\lambda}\right)_{k}\left(q^{1-\lambda}\right)_{k} .
$$

2. Cyclotomic completions of polynomial rings. We present and modify some results of [6] here. Let $R$ be a commutative ring with unit, and let $R[q]$ be the polynomial ring over $R$. Recall that $\Phi_{n}(q)$ denotes the $n$th cyclotomic polynomial. If $S \subset \mathbb{N}$, we set $\Phi_{S}=\left\{\Phi_{n}(q) \mid n \in S\right\}$. Let $\Phi_{S}^{*}$ denote the multiplicative set in $\mathbb{Z}[q]$ generated by $\Phi_{S}$ and directed with 
respect to the divisibility relation. The principal ideals $(f(q)) \subset R[q]$ for $f(q) \in \Phi_{S}^{*}$ define a linear topology of the ring $R[q]$. In [6], Habiro defined the $(S)$-cyclotomic completion ring $R[q]^{S}$ as follows:

$$
R[q]^{S}:=\lim _{f(q) \in \Phi_{S}^{*}} \frac{R[q]}{(f(q))} .
$$

For example, since the sequence $(q)_{n \in \mathbb{N}}$ is cofinal in $\Phi_{\mathbb{N}}^{*}$, we have

$$
\widehat{\mathbb{Z}[q]} \simeq \mathbb{Z}[q]^{\mathbb{N}} \text {. }
$$

Similarly, for $S=\{n \in \mathbb{N} \mid n \not \equiv 2(\bmod 4)\}$,

$$
\widehat{\mathbb{Z}[v]_{2}} \simeq \mathbb{Z}[v]^{S}
$$

Note that if $S$ is finite, then $R[q]^{S}$ is identified with the $\left(\prod \Phi_{S}\right)$-adic completion of $R[q]$. In particular,

$$
R[q]^{\{1\}} \simeq R[[q-1]], \quad R[q]^{\{2\}} \simeq R[[q+1]] .
$$

If $S^{\prime} \subset S$, then $\Phi_{S^{\prime}}^{*} \subset \Phi_{S}^{*}$, hence there is a natural map

$$
\varrho_{S, S^{\prime}}^{R}: R[v]^{S} \rightarrow R[v]^{S^{\prime}}
$$

In particular, when $S=\{n \in \mathbb{N} \mid n \not \equiv 2(\bmod 4)\}$ and $S^{\prime}=\{1\}$, the map

$$
\varrho_{S, S^{\prime}}^{\mathbb{Z}}: \widehat{\mathbb{Z}[v]_{2}} \rightarrow \mathbb{Z}[[v-1]]
$$

is the Taylor expansion.

Recall important results concerning $R[v]^{S}$ from [6]. Two positive integers $n, n^{\prime}$ are called adjacent if $n^{\prime} / n=p^{e}$ with $e \in \mathbb{Z}$ and a prime $p$ such that the ring $R$ is $p$-adically separated. A set of positive integers is connected if for any two distinct elements $n, n^{\prime}$ there is a sequence $n=n_{1}, n_{2}, \ldots, n_{k-1}, n_{k}=$ $n^{\prime}$ in the set such that any two consecutive numbers of this sequence are adjacent. Theorem 4.2 of [6] says that if $S$ is connected, then for any subset $S^{\prime} \subset S$, the natural map $\varrho_{S, S^{\prime}}^{R}: R[v]^{S} \rightarrow R[v]^{S^{\prime}}$ is an embedding.

If $\zeta$ is a root of unity of order in $S$, then for every $f(v) \in R[v]^{S}$ the evaluation $\operatorname{ev}_{\zeta}(f(v)) \in R[\xi]$ can be defined by sending $v \mapsto \zeta$. For a set $\Xi$ of roots of unity whose orders form a subset $\mathcal{T} \subset S$, one defines the evaluation

$$
\mathrm{ev} \Xi: R[v]^{S} \rightarrow \prod_{\zeta \in \Xi} R[\zeta] .
$$

Theorem 6.1 of [6] shows that if $R \subset \mathbb{Q}$ and $S$ is connected, and there exists $n \in S$ that is adjacent to infinitely many elements in $\mathcal{T}$, then ev $\Xi$ is injective.

Proposition 2.1.

(a) The Taylor expansion map $T: \widehat{\mathbb{Z}[v]_{2}} \rightarrow \mathbb{Z}[[v-1]]$ is injective. 
(b) For a non-negative integer $k \neq 1$ and an odd prime number $p$, let $\mathcal{T}_{k}=\left\{2^{k} p^{e} \mid e \in \mathbb{N}\right\}$. Suppose $f(v), g(v) \in \widehat{\mathbb{Z}[v]_{2}}$ are such that $f(\zeta)=$ $g(\zeta)$ for every $\zeta$ with $\operatorname{ord}(\zeta) \in \mathcal{T}_{k}$. Then $f(v)=g(v)$.

(c) The natural Taylor map $\widehat{\mathbb{Z}[v]} \rightarrow \mathbb{Z}[I, 1 / 2][[1+q]]$, explained in the proof, is injective. Here $I$ is the imaginary unit, $I^{2}=-1$.

Proof. (a) It is easy to see that $S=\{n \in \mathbb{N} \mid n \not \equiv 2(\bmod 4)\}$ is connected. Note that if $S^{\prime}=\{1\}$ then $\mathbb{Z}[v]^{S^{\prime}}=\mathbb{Z}[[v-1]]$. Hence part (a) follows from the above mentioned Theorem 4.2 of [6].

(b) Since $k \neq 1,2^{k} \in S$, and by assumption, $2^{k}$ is adjacent to every element in $\mathcal{T}$. Now (b) follows from the above mentioned [6, Theorem 6.1].

(c) It is easy to see that the set $S=\{n \in \mathbb{N} \mid n \equiv 0(\bmod 4)\}$ is connected, and $\widehat{\mathbb{Z}[v]_{S}} \cong \mathbb{Z}[v]^{S}$. Hence $\varrho_{S,\{4\}}^{\mathbb{Z}}$ is injective. For the set $\{4\}$, we have

$$
\mathbb{Z}[v]^{\{4\}} \simeq \mathbb{Z}[v]\left[\left[1+v^{2}\right]\right] \simeq \mathbb{Z}[v][[1+q]]
$$

Using

$$
v=\sqrt{q}=\sqrt{-(1-(1+q))}=I(1-(1+q))^{1 / 2} \in \mathbb{Z}[I][1 / 2][[1+q]]
$$

we see that there is an embedding of $\mathbb{Z}[v]^{\{4\}}$ into $\mathbb{Z}[I, 1 / 2][[1+q]]$, which, combined with $\varrho_{S,\{4\}}^{\mathbb{Z}}$, gives us the injective Taylor map.

Let $S_{k}=\left\{2^{k}(2 n+1) \mid n \in \mathbb{N}\right\}$. Then for every $k \geq 2$ and $f(v) \in$ $\mathbb{Z}[1 / 2, I][v]^{S_{k}}$, and any root of unity $\zeta$ of order in $S_{k}$, the evaluation map ev $\operatorname{ev}_{\zeta}$ : $\mathbb{Z}[1 / 2, I][v]^{S} \rightarrow \mathbb{Z}[1 / 2][\zeta]$ can be defined as follows: $\operatorname{ev}_{\zeta}(v)=\zeta, \operatorname{ev}_{\zeta}(I)=$ $\zeta^{\operatorname{ord}(\zeta) / 4}$.

Let us study, for $\Gamma:=\mathbb{Z}[1 / 2][v]$ and $S=\{n \in \mathbb{N} \mid n \not \equiv 2(\bmod 4)\}$, the completion $\Gamma^{S}$ mentioned in the introduction. Note that $S$ is not connected in Habiro's sense for $R=\mathbb{Z}[1 / 2]$. We have $S=\bigcup_{k \in \mathbb{N}_{0}, k \neq 1} S_{k}$ with $\mathbb{N}_{0}=$ $\{0,1,2, \ldots\}$.

Proposition 2.2.

(a) One has

$$
\Gamma^{S}=\prod_{k \in \mathbb{N}_{0}, k \neq 1} \Gamma^{S_{k}}
$$

(b) For an integer $k \geq 2$ and an odd prime number $p$, let $\mathcal{T}_{k}=\left\{2^{k} p^{e} \mid\right.$ $e \in \mathbb{N}\}$. Suppose $f(v), g(v) \in \mathbb{Z}[1 / 2, I][v]^{S_{k}}$ are such that $\operatorname{ev}_{\zeta}(f(v))=$ $\operatorname{ev}_{\zeta}(g(v))$ for every $\zeta$ with $\operatorname{ord}(\zeta) \in \mathcal{T}_{k}$. Then $f(v)=g(v)$.

(c) For an odd prime number $p$, let $\mathcal{T}=\left\{2^{k} p^{e} \mid k, e \in \mathbb{N}\right\}$. Suppose $f(v), g(v) \in \Gamma^{S}$ are such that $\operatorname{ev}_{\zeta}(f(v))=\operatorname{ev}_{\zeta}(g(v))$ for every $\zeta$ with $\operatorname{ord}(\zeta) \in \mathcal{T}$. Then $f(v)=g(v)$.

Proof. (a) Let us first prove that if $n=2^{k} n^{\prime} \in S_{k}$ and $m=2^{l} m^{\prime} \in S_{l}$ with $k \neq l$, then $\left(\Phi_{n}, \Phi_{m}\right)=(1)$ in $\Gamma=\mathbb{Z}[1 / 2][v]$. Indeed, if $n^{\prime} \neq m^{\prime}$, then $n$ 
and $m$ are not adjacent, hence $\left(\Phi_{n}, \Phi_{m}\right)=(1)$ in $\mathbb{Z}[v]$ and the claim holds. If $n^{\prime}=m^{\prime}$, then $n / m=2^{k-l}$, hence in $\mathbb{Z}[v]$ one has $2 \in\left(\Phi_{n}, \Phi_{m}\right)$, which implies the claim, since 2 is invertible in $\Gamma$.

If $f \in \Phi_{S}^{*}$, then $f=\prod f_{k}$ with $f_{k} \in \Phi_{S_{k}}^{*}$. Hence the $f_{k}$ 's are pairwise coprime. By the Chinese remainder theorem,

$$
\frac{\Gamma}{(f)}=\prod_{k} \frac{\Gamma}{\left(f_{k}\right)} .
$$

Taking the inverse limit, we get (a).

(b) It is easy to see that $S_{k}$ is connected in Habiro's sense for the ring $\mathbb{Z}[1 / 2, I]$. Hence part (b) follows by adapting the proof of $[6$, Theorem 6.1$]$ to the ring $\mathbb{Z}[1 / 2, I]$, which is straightforward.

(c) is an easy consequence of (a) and (b).

3. Laplace transform. In this section we introduce the Laplace transform method. For simplicity, we restrict to homology spheres obtained by surgery on knots; the general case can be obtained by applying the same arguments to each component of the link.

3.1. Quantum invariants for knot surgeries. For any non-zero integer $b$, and a knot $K$, let $M=S^{3}\left(K_{b}\right)$ be a homology sphere obtained by surgery on $K$ with framing $b$. Assume that $\xi$ is a primitive $r$ th root of unity and $r$ is even. The quantum $\mathrm{SU}(2)$ invariant of $M$ is defined as follows:

$$
\tau_{M}(\xi)=\frac{\sum_{\lambda}^{\xi} q^{b\left(\lambda^{2}-1\right) / 4}\left(1-q^{\lambda}\right)\left(1-q^{-\lambda}\right) J_{K}^{\prime}(\lambda)}{\sum_{\lambda}^{\xi} q^{\operatorname{sn}(b)\left(\lambda^{2}-1\right) / 4}\left(1-q^{\lambda}\right)\left(1-q^{-\lambda}\right)},
$$

where $\operatorname{sn}(b)$ is the sign of $b$. To be precise, one needs to fix a 4 th root of $\xi$. Note that when computing the Jones polynomial of a knot (or a link) further in this paper, we always assume that its framing is zero. However, in the formula for the quantum invariant, framing is taken into account by means of the factor $q^{b\left(\lambda^{2}-1\right) / 4}$.

Substituting Habiro's formula (5) into (7) we get

$$
\tau_{M}(\xi)=\frac{\sum_{\lambda}^{\xi} q^{b\left(\lambda^{2}-1\right) / 4} \sum_{n=0}^{\infty} C_{K, n} F_{n}\left(q^{\lambda}, q\right)}{\sum_{\lambda}^{\xi} q^{\operatorname{sn}(b)\left(\lambda^{2}-1\right) / 4} F_{0}\left(q^{\lambda}, q\right)},
$$

where $F_{n}\left(q^{\lambda}, q\right)=\left(q^{\lambda}\right)_{n+1}\left(q^{-\lambda}\right)_{n+1}$.

Suppose $r$ is odd. Then, taking the sums over odd $\lambda$ in the numerator and the denominator of $(7)$ we get $\tau_{M}^{\mathrm{SO}(3)}(\xi)$. In this case, there is no need to fix a 4 th root of $\xi$.

3.2. Laplace transform method. The main idea behind the Laplace transform method is to interchange the sums over $\lambda$ and $n$ in (8) and regard $\sum_{\lambda}^{\xi} q^{b\left(\lambda^{2}-1\right) / 4}$ as an operator (called Laplace transform) acting on $F_{n}\left(q^{\lambda}, q\right)$. 
(Recall that $\int e^{-a x^{k}} f(x) d x$ is called the Laplace transform of $f$ of order $k$. Our sum is a discrete version of the Laplace transform of the second order.)

More precisely, after interchanging the sums in the numerator of (8) we get

$$
\sum_{n=0}^{r-1} C_{K, n}(q) \sum_{\lambda}^{\xi} q^{b\left(\lambda^{2}-1\right) / 4} F_{n}\left(q^{\lambda}, q\right) .
$$

Now observe that $F_{n}\left(q^{\lambda}, q\right)=\left(q^{\lambda}\right)_{n+1}\left(q^{-\lambda}\right)_{n+1}$ is a Laurent polynomial in two variables $q^{\lambda}$ and $q$. The Laplace transform does not affect $q$, and we only need to compute the action of the Laplace operator on $q^{a \lambda}$.

Suppose $b=1,2$ and $r$ is even. A simple square completion argument shows that

$$
\sum_{\lambda}^{\xi} q^{b\left(\lambda^{2}-1\right) / 4} q^{a \lambda}=\xi^{-a^{2} /(b, r)} \gamma_{b}(\xi) .
$$

Summarizing the previous discussion, we get

$$
\sum_{\lambda}^{\xi} q^{b\left(\lambda^{2}-1\right) / 4} F_{n}\left(q^{\lambda}, q\right)=\operatorname{ev}_{\xi}\left(L_{b ; \lambda}\left(F_{n}\left(q^{\lambda}, q\right)\right)\right) \gamma_{b}(\xi) .
$$

Here $L_{b ; \lambda}(F)$ is the Laplace transform of $F$, which is defined as follows. Suppose $F$ is a formal power series in $q^{ \pm 1}$ and $q^{ \pm \lambda}$. Then $L_{b ; \lambda}(F)$ is obtained from $F$ by replacing every $q^{a \lambda}$ by $q^{-a^{2} / b}$. The evaluation map ev $\xi$ converts $q^{1 / b}$ to $\xi^{1 /(b, r)}$. Note that while ev $\mathrm{v}_{\xi}$ might depend on $r$, the Laplace transform $L_{b ; \lambda}$ does not.

If $r$ is odd and $(b, r)=1$, we can define the Laplace transform $L_{b ; \lambda}$ : $\mathbb{Z}\left[q^{ \pm \lambda}, q^{ \pm 1}\right] \rightarrow \mathbb{Z}\left[q^{ \pm 1 / b}\right]$ as the $\mathbb{Z}\left[q^{ \pm 1}\right]$-linear operator sending $q^{a \lambda} \mapsto q^{-a^{2} / b}$. In this case, we have

$$
\sum_{\lambda}^{\xi, 1} q^{b\left(\lambda^{2}-1\right) / 4} F_{n}\left(q^{\lambda}, q\right)=\operatorname{ev}_{\xi}\left(L_{b ; \lambda}\left(F_{n}\left(q^{\lambda}, q\right)\right)\right) \gamma_{b}^{1}(\xi) .
$$

As a result, we have closed formulas for quantum invariants in terms of the Laplace transform.

Theorem 3.1. Let $M=S^{3}\left(K_{b}\right)$ and $b=1$ or 2 . Then

$$
\begin{aligned}
\tau_{M}(\xi) & =\frac{1}{2\left(1-\xi^{-\operatorname{sn}(b)}\right)} \frac{\gamma_{b}(\xi)}{\gamma_{\mathrm{sn}(b)}(\xi)} \sum_{n=0}^{\infty} C_{K, n} \mathrm{ev}_{\xi}\left(L_{b ; \lambda}\left(F_{n}\right)\right), \\
\tau_{M}^{\mathrm{SO}(3)}(\xi) & =\frac{1}{2\left(1-\xi^{-\operatorname{sn}(b)}\right)} \frac{\gamma_{b}^{1}(\xi)}{\gamma_{\mathrm{sn}(b)}^{1}(\xi)} \sum_{n=0}^{\infty} C_{K, n} \mathrm{ev}_{\xi}\left(L_{b ; \lambda}\left(F_{n}\right)\right) .
\end{aligned}
$$

Note that the second formula holds for any $b$ coprime to the order of $\xi$ (cf. in [10], [2]). In the case when $(b, \operatorname{ord}(\xi)) \neq 1$, the formulas for quantum $\mathrm{SO}(3)$ invariants are given in [1]. 
4. Habiro theory. In this section we show how Theorem 3.1 can be used to compute Habiro's unified invariant of integral homology spheres.

4.1. Knot surgeries. Any knot surgery with framing $b= \pm 1$ yields an integral homology sphere. Combining Theorem 3.1 with Lemma 4.2 below we get the following theorem.

Theorem 4.1 (Habiro). For $M_{ \pm}=S^{3}\left(K_{ \pm 1}\right)$, there exists a unique invariant

$$
\begin{aligned}
& I_{M_{+}}(q)=\sum_{n=0}^{\infty}(-1)^{n} q^{-n(n+3) / 2} C_{K, n}(q) \frac{\left(q^{n+1}\right)_{n+1}}{1-q} \in \widehat{\mathbb{Z}[q]}, \\
& I_{M_{-}}(q)=\sum_{n=0}^{\infty} C_{K, n}(q) \frac{\left(q^{n+1}\right)_{n+1}}{1-q} \in \widehat{\mathbb{Z}[q]}
\end{aligned}
$$

such that $I_{M_{ \pm}}(\xi)=\tau_{M_{ \pm}}(\xi)=\tau_{M_{ \pm}}^{\mathrm{SO}(3)}(\xi)$.

Examples. Denote by $S^{3}\left(3_{1}\right)$ and $S^{3}\left(4_{1}\right)$ the Poincaré sphere and the 3 -manifold obtained by framing 1 surgery on the figure 8 knot. By Theorem 4.1, we have

$$
\begin{aligned}
& I_{S^{3}\left(3_{1}\right)}(q)=\frac{q}{1-q} \sum_{k=0}^{\infty}(-1)^{k} q^{-(k+2)(3 k+1) / 2}\left(q^{k+1}\right)_{k+1}, \\
& I_{S^{3}\left(4_{1}\right)}(q)=\frac{q}{1-q} \sum_{k=0}^{\infty}(-1)^{k} q^{-(k+1)^{2}}\left(q^{k+1}\right)_{k+1} .
\end{aligned}
$$

\section{LEMMA 4.2.}

$$
\begin{gathered}
L_{-1 ; \lambda}\left(\left(q^{\lambda}\right)_{k+1}\left(q^{-\lambda}\right)_{k+1}\right)=2\left(q^{k+1}\right)_{k+1}, \\
L_{1 ; \lambda}\left(\left(q^{\lambda}\right)_{k+1}\left(q^{-\lambda}\right)_{k+1}\right)=2(-1)^{k+1} q^{-(k+2)(k+1) / 2}\left(q^{k+1}\right)_{k+1} .
\end{gathered}
$$

Proof. Let $L_{b ; \lambda}: \mathbb{Z}\left[q^{ \pm \lambda}, q^{ \pm 1}\right] \rightarrow \mathbb{Z}\left[q^{ \pm 1 / b}\right]$ be the $\mathbb{Z}\left[q^{ \pm 1}\right]$-linear operator sending $q^{a \lambda} \mapsto q^{-a^{2} / b}$. Then for $F_{k}\left(q^{\lambda}, q\right) \in \mathbb{Z}\left[q^{ \pm \lambda}, q^{ \pm 1}\right]$ we have

$$
L_{-b ; \lambda}\left(F_{k}\left(q^{\lambda}, q\right)\right)=q^{k(k+1)} L_{b ; \lambda}\left(F\left(q^{\lambda}, q^{-1}\right)\right) .
$$

Using this formula we can deduce (10) from (9).

Let us prove (9). For this, we split

$$
F_{k}\left(q^{\lambda}, q\right)=S_{k}\left(q^{\lambda}, q\right)+T_{k}\left(q^{\lambda}, q\right)
$$

with $S_{k}\left(q^{\lambda}, q\right)=\left(q^{\lambda}\right)_{k+1}\left(q^{-\lambda+1}\right)_{k}$ and $T_{k}\left(q^{\lambda}, q\right)=-q^{-\lambda}\left(q^{\lambda}\right)_{k+1}\left(q^{-\lambda+1}\right)_{k}$. Then $S_{k}\left(q^{-\lambda}, q\right)=T_{k}\left(q^{\lambda}, q\right)$ implies $L_{b ; \lambda}\left(S_{k}\right)=L_{b ; \lambda}\left(T_{k}\right)$ for any $b$. Therefore, we have to look at one of them only. 
Further, by the $q$-binomial theorem (eq. (II.4) in [4]) we get

$$
\begin{aligned}
S_{k}\left(q^{\lambda}, q\right) & =(-1)^{k} q^{-k \lambda} q^{k(k+1) / 2}\left(q^{\lambda-k}\right)_{2 k+1} \\
& =(-1)^{k} q^{k(k+1) / 2} \sum_{j=0}^{2 k+1}(-1)^{j}\left[\begin{array}{c}
2 k+1 \\
j
\end{array}\right]_{q} q^{j(j-1) / 2} q^{-k j} q^{(j-k) \lambda},
\end{aligned}
$$

where

$$
\left[\begin{array}{l}
n \\
k
\end{array}\right]_{q}=\frac{(q)_{n}}{(q)_{k}(q)_{n-k}} .
$$

Taking the Laplace transform we have

$$
L_{-1 ; \lambda}\left(S_{k}\left(q^{\lambda}, q\right)\right)=(-1)^{k} q^{\left(3 k^{2}+k\right) / 2} \sum_{j=o}^{2 k+1} \frac{\left(q^{-2 k-1}\right)_{j}}{(q)_{j}} q^{j^{2}+j-j k} .
$$

The result now follows by applying the Sears-Carlitz transformation (eq. (III.14) in [4]) for terminating ${ }_{3} \phi_{2}$ series with specializations $a=q^{-2 k-1}$, $b, c \rightarrow \infty, z \rightarrow q^{k+2}$.

4.2. Link surgeries. Let $M$ be an integral homology sphere. Without loss of generality, we can assume that $M$ is obtained by surgery on an algebraically split link $L$ in $S^{3}$ with framings \pm 1 . Suppose that the first $\sigma_{+}$ components have framing +1 , and the others -1 . Substituting the cyclotomic expansion of the colored Jones polynomial (given in Proposition 1.1) into (1.3) and applying the Laplace transform method to each component of $L$, we derive the following formula for the unified invariant of $M$.

Theorem 4.3 (Habiro). For $M$ as above, there exists a unique invariant

$$
I_{M}(q)=\sum_{k=0}^{\infty}\left(\sum_{\max k_{i}=k} C_{L, \mathbf{k}}(v) \prod_{i=1}^{\sigma_{+}}(-1)^{k_{i}} q^{-k_{i}\left(k_{i}+3\right) / 2}\right) \frac{\left(q^{k+1}\right)_{k+1}}{1-q} \in \widehat{\mathbb{Z}[q]}
$$

such that $I_{M}(\xi)=\tau_{M}(\xi)=\tau_{M}^{\mathrm{SO}(3)}(\xi)$.

5. Rational homology 3-spheres with $H_{1}(M)=(\mathbb{Z} / 2 \mathbb{Z})^{n}$. In this section we define the unified invariant for $M \in \mathcal{M}_{n}$.

5.1. Normalization. Suppose that the order of $\zeta$ is divisible by 4 . It is easy to show that the quantum invariant of $\mathbb{R} P^{3}$, which is obtained by surgery on the unknot with framing 2 , is given by

$$
\tau_{\mathbb{R} P^{3}}(\xi)=\frac{\gamma_{2}(\xi)}{\left(1+\zeta^{-1}\right) \gamma_{1}(\xi)}=\frac{\gamma_{-2}(\xi)}{(1+\zeta) \gamma_{-1}(\xi)}=\frac{\zeta^{-1 / 2} \sqrt{2}}{1+\zeta^{-1}} .
$$


For $M \in \mathcal{M}_{n}$, we will use a normalization such that the connected sum of $n$ projective spaces $\mathbb{R} P^{3}$ takes value 1 :

$$
\tau_{M}^{\prime}(\xi):=\frac{\tau_{M}(\xi)}{\left(\tau_{\mathbb{R} P^{3}}(\xi)\right)^{n}} .
$$

For an odd root of unity $\xi$, we put

$$
\tau_{M}^{\prime}(\xi):=\frac{\tau_{M}^{\mathrm{SO}(3)}(\xi)}{\left(\tau_{\mathbb{R} P^{3}}^{\mathrm{SO}(3)}(\xi)\right)^{n}} \quad \text { with } \quad \tau_{\mathbb{R} P^{3}}^{\mathrm{SO}(3)}(\xi)=\frac{\gamma_{2}^{1}(\xi)}{\left(1+\zeta^{-1}\right) \gamma_{1}^{1}(\xi)} .
$$

5.2. Diagonalization. Recall that a linking pairing on a finite Abelian group $G$ is a non-singular symmetric bilinear map from $G \times G$ to $\mathbb{Q} / \mathbb{Z}$. Two linking pairings $\nu, \nu^{\prime}$ on respectively $G, G^{\prime}$ are equivalent if there is an isomorphism between $G$ and $G^{\prime}$ carrying $\nu$ to $\nu^{\prime}$. With the obvious block sum, the set of equivalence classes of linking pairings is a semigroup.

One type of linking pairing is given by non-singular square symmetric matrices with integer entries: any such $n \times n$ matrix $A$ gives rise to a linking pairing $\phi(A)$ on $G=\mathbb{Z}^{n} / A \mathbb{Z}^{n}$ defined by $\phi(A)\left(v, v^{\prime}\right)=v^{t} A^{-1} v^{\prime} \in \mathbb{Q} \bmod \mathbb{Z}$, where $v, v^{\prime} \in \mathbb{Z}^{n}$. If there is a diagonal matrix $A$ such that a linking pairing $\nu$ is isomorphic to $\phi(A)$, then we say that $\nu$ is of diagonal type. It is known that if the linking pairing of a 3-manifold is of diagonal type, with diagonal entries $d_{1}, \ldots, d_{k}$, then $M$ can be obtained by surgery along an algebraically split link, with framings $d_{1}, \ldots, d_{k}$ on $k$ components, and \pm 1 on the others (see $[18,10])$.

Lemma 5.1. Suppose $H_{1}(M, \mathbb{Z})=(\mathbb{Z} / 2 \mathbb{Z})^{n}$. Then $M \# \mathbb{R} P^{3}$ can be obtained from $S^{3}$ by surgery on an algebraically split link with framing 2 on $n+1$ components, and \pm 1 on the others.

Proof. The generators of the semigroup of linking pairings are known (see $[8,20])$. Since subgroups of $H_{1}$ are of the form $(\mathbb{Z} / 2 \mathbb{Z})^{n}$ only, from the list of generators we see that a linking pairing $\phi$ on $H_{1}(M, \mathbb{Z})$ must be the block sum of linking pairings, each of the form $\phi(2)$ or $E_{0}^{1}$ in the notation of [8]. Here $\phi(2)$ is considered as the $1 \times 1$ matrix with the only entry 2 , and $E_{0}^{1}$ is a linking pairing on $\mathbb{Z} / 2 \mathbb{Z} \times \mathbb{Z} / 2 \mathbb{Z}$.

Note that $\phi(2)$ is the linking pairing of $\mathbb{R} P^{3}$. One of the relations among generators says that $E_{0}^{1} \oplus \phi(2)=\phi(2) \oplus \phi(2) \oplus \phi(2)$. Thus, after adding a copy of $\mathbb{R} P^{3}$ the linking pairing of $M$ is of diagonal type, with entries 2 on the diagonal, and we get the result.

5.3. Unified invariant. For any $M \in \mathcal{M}_{n}$, by Lemma $5.1, M^{\prime}=M \# \mathbb{R} P^{3}$ can be obtained by surgery on an algebraically split $m$-component link $L$. Let us assume that the first $s_{+}$components of $L$ have framing 2 , the next $s_{-}$ 
components have framing -2 , the next $l_{+}$components are 1 -framed, and the last components are $(-1)$-framed. Although we can avoid the -2 framing, we add it for the convenience of calculation. Note that $s_{+}+s_{-}=n+1$, since $M^{\prime} \in \mathcal{M}_{n+1}$. The unified invariant of $M$ is defined by

$$
\begin{aligned}
& I_{M}(v)=\frac{(1+v)^{n+1}}{1-q} \sum_{k \geq 0}\left(q^{k+1}\right)_{k+1} \\
& \times\left(\sum_{\max k_{i}=k} C_{L, \mathbf{k}}(v) \prod_{i=1}^{s_{+}}(-v)^{-k_{i}} B_{k_{i}}(v) \prod_{i=s_{+}+1}^{n+1} B_{k_{i}}(v) \prod_{i=n+2}^{n+1+l_{+}}(-1)^{k_{i}} q^{-k_{i}\left(k_{i}+3\right) / 2}\right),
\end{aligned}
$$

where $B_{k}(v)=\left(\prod_{i=0}^{k}\left(1+v^{2 i+1}\right)\right)^{-1}$.

5.4. Proof of Theorem 2. Let us first show that $I_{M}(v) \in \Gamma^{S}$. Indeed, the denominator of $I_{M}$ contains only $\Phi_{4 i+2}(v)$ for $i \in \mathbb{N}$, which are invertible modulo $\Phi_{s}(v)$ for any $s \in S$ in $\mathbb{Z}[1 / 2][v]$ (cf. the proof of Proposition 2.2(a)).

We next show that $\operatorname{ev}_{\xi}\left(I_{M}(v)\right)=\tau_{M^{\prime}}^{\prime}(\xi)$. Observe that $\tau_{M^{\prime}}^{\prime}(\xi)=\tau_{M}^{\prime}(\xi)$ by the definition. The proof is an application of the Laplace transform method. Using Lemma 5.2 below we have

$$
\begin{aligned}
\operatorname{ev}_{\xi}\left(\frac{L_{2 ; \lambda}\left[\left(q^{\lambda}\right)_{k+1}\left(q^{-\lambda}\right)_{k+1}\right]}{2\left(1-q^{-1}\right)}\right) \frac{\gamma_{2}(\xi)}{\gamma_{1}(\xi)} & =\operatorname{ev}_{\xi}\left((-v)^{-k}\left(-v^{2} ;-v\right)_{2 k}\right) \tau_{\mathbb{R} P^{3}}(\xi), \\
\operatorname{ev}_{\xi}\left(\frac{L_{-2 ; \lambda}\left[\left(q^{\lambda}\right)_{k+1}\left(q^{-\lambda}\right)_{k+1}\right]}{2(1-q)}\right) \frac{\gamma_{-2}(\xi)}{\gamma_{-1}(\xi)} & =\operatorname{ev}_{\xi}\left(\left(-v^{2} ;-v\right)_{2 k}\right) \tau_{\mathbb{R} P^{3}}(\xi) .
\end{aligned}
$$

In addition, we use the following identity:

$$
\frac{(1-v)\left(-v^{2} ;-v\right)_{2 k}}{\left(q^{k+1}\right)_{k+1}}=B_{k}(v),
$$

whose proof is left to the reader. Uniqueness of $I_{M}$ follows from Proposition $2.2(\mathrm{c})$.

\section{LEMMA 5.2.}

$$
\begin{aligned}
L_{-2 ; \lambda}\left(F_{k}\left(q^{\lambda}, q\right)\right) & =2(1-v)\left(-v^{2} ;-v\right)_{2 k}, \\
L_{2 ; \lambda}\left(F_{k}\left(q^{\lambda}, q\right)\right) & =2(-1)^{k+1} v^{-k-1}(1-v)\left(-v^{2} ;-v\right)_{2 k} .
\end{aligned}
$$

Proof. Recall that $L_{ \pm 2 ; \lambda}\left(q^{a \lambda}\right)=v^{\mp a^{2}}$. We proceed by proving the first formula. By the $q$-binomial theorem we get

$$
L_{-2 ; \lambda}\left(F_{k}\left(q^{\lambda}, q\right)\right)=2(-1)^{k} q^{k^{2}+k / 2} \sum_{j=0}^{2 k+1} \frac{\left(q^{-2 k-1}\right)_{j}}{(q)_{j}} q^{j+j^{2} / 2} .
$$

The Sears-Carlitz transformation (eq. (III.14) in [4]) with $a=q^{-2 k-1}, c=-q^{-k}$, $z=q^{k+3 / 2}$ and $b \rightarrow \infty$ reduces this sum to ${ }_{2} \phi_{1}\left(-q^{-k-1 / 2}, q^{-k} ; q^{-k+1 / 2}, q\right)$, which can be computed by the $q$-Vandermonde formula (eq. (II.6) in [4]), 
and the result follows. Note that $L_{2 ; \lambda}$ can be computed from $L_{-2 ; \lambda}$ by the same argument as in the proof of Lemma 4.2.

Proof of Corollary 3. Part (a) follows, since $\operatorname{ev}_{\zeta}\left(\Gamma^{S}\right)=\mathbb{Z}[1 / 2][\zeta]$ if $\operatorname{ord}(\zeta) \in S$. Part (b) is a direct consequence of Proposition 2.2(c).

5.5. Case $\left|H_{1}\right|=2$. It is well-known that any $M \in \mathcal{M}_{1}$ can be obtained by surgery on an algebraically split link $L$. Let us assume that $L_{ \pm}$is a link of $m+1$ components numbered by $0,1, \ldots, m$, where the 0 th component has framing \pm 2 , the next $s$ components have framing 1 , and the remaining ones have framing -1 . Assume $M_{ \pm}=S^{3}\left(L_{ \pm}\right)$. The following proposition is a direct consequence of Theorem 2 and implies the part of Theorem 6 concerning the non-refined invariants.

Proposition 5.3. For $M_{ \pm}$as above, the unified invariants $I_{M_{ \pm}}$belong to $\widehat{\mathbb{Z}[v}_{2}$ and are given by the following formulas:

$$
\begin{aligned}
I_{M_{+}}(v)=\sum_{k_{i} \geq 0}\left(\sum_{\max k_{i}=k} C_{L, \mathbf{k}}(v)(-v)^{-k_{0}}\right. & \\
& \left.\times \prod_{i=k_{0}+1}^{k}\left(1+v^{2 i+1}\right) \prod_{i=1}^{s}(-1)^{k_{i}} q^{-k_{i}\left(k_{i}+3\right) / 2}\right)\left(-v^{2} ;-v\right)_{2 k}
\end{aligned}
$$

and

$$
\begin{aligned}
I_{M_{-}}(v)=\sum_{k_{i} \geq 0}\left(\sum_{\max k_{i}=k} C_{L, \mathbf{k}}(v)\right. & \\
& \left.\times \prod_{i=k_{0}+1}^{k}\left(1+v^{2 i+1}\right) \prod_{i=1}^{s}(-1)^{k_{i}} q^{-k_{i}\left(k_{i}+3\right) / 2}\right)\left(-v^{2} ;-v\right)_{2 k} .
\end{aligned}
$$

ExAmPLE. Let $L$ be the Whitehead link with framings 2 and -1 and $M=S^{3}(L)$. Then

$$
I_{M}(v)=\sum_{k=0}^{\infty} v^{-k(k+2)}\left(-v^{2} ;-v\right)_{2 k} .
$$

5.6. Proof of Corollary \%. (a) follows from the fact that $\tau_{M}^{\prime}(\xi)=\operatorname{ev}_{\xi}\left(I_{M}\right)$, and for any $f \in \widehat{\mathbb{Z}[v]_{2}}, \operatorname{ev}_{\xi}(f) \in \mathbb{Z}[\zeta]$.

(b) An infinite set of roots of unity of orders odd prime powers is a set of the form $\mathcal{T}_{k}$ in Proposition 2.1(b). Hence Proposition 2.1(b) implies the result.

(c) The Ohtsuki series is just the Taylor expansion of $I_{M}$. Observe that the Taylor series in $1-v$ can be converted into a formal power series in $1-q$ by 


$$
v-1=(1+(q-1))^{1 / 2}-1=\sum_{n=1}^{\infty}\left(\begin{array}{c}
1 / 2 \\
n
\end{array}\right)(q-1)^{n},
$$

where $\left(\begin{array}{c}1 / 2 \\ n\end{array}\right) \in \mathbb{Z}[1 / 2]$.

(d) The LMO invariant determines the Ohtsuki series via the $\mathrm{sl}_{2}$ weight system (see [18]).

6. Refinements. In this section we show that the Laplace transform method can effectively be used to define refinements of the unified invariant.

6.1. Spin case. Suppose $M \in \mathcal{M}_{n}$ can be obtained by surgery on an algebraically split $m$ component link $L$ as described in Section 5.3, where the first $n$ components of $L$ are $( \pm 2)$-framed, and the remaining components are $( \pm 1)$-framed. Suppose $\sigma_{c}$ is a spin structure on $M$ corresponding to the solution $c$ of the characteristic equation. There are $2^{n}$ spin structures on $M$.

Let

$$
B_{k}(x, v)=\frac{1}{2}\left(\prod_{i=0}^{k}\left(1-v^{2 i+1}\right)+x \prod_{i=0}^{k}\left(1+v^{2 i+1}\right)\right) \prod_{i=0}^{k} \frac{1}{1-q^{2 i+1}} .
$$

Then we define the unified invariant of $\left(M, \sigma_{c}\right)$ as follows:

$$
\begin{aligned}
& I_{M, \sigma_{c}}(v)=\frac{(1+v)^{n}}{1-q} \sum_{k \geq 0}\left(q^{k+1}\right)_{k+1}\left(\sum_{\max k_{i}=k} C_{L, \mathbf{k}}(v) \prod_{i=1}^{s_{+}}(-v)^{-k_{i}}\right. \\
& \left.\times B_{k_{i}}\left((-1)^{c_{i}+k_{i}}, v\right) \prod_{i=s_{+}+1}^{n} B_{k_{i}}\left((-1)^{c_{i}+1}, v\right) \prod_{i=n+1}^{n+l_{+}}(-1)^{k_{i}} q^{-k_{i}\left(k_{i}+3\right) / 2}\right) .
\end{aligned}
$$

More generally, $M^{\prime}=M \# \mathbb{R} P^{3}$ can be obtained by surgery on an algebraically split link, i.e. $I_{M^{\prime}, \sigma^{\prime}}$ is defined by (12). Assume that $\sigma^{\prime}$ and $\sigma^{\prime \prime}$ are the two spin structures on $M^{\prime}$ whose restrictions to $M$ coincide with $\sigma$. Then we define the unified invariant of $(M, \sigma)$ as follows:

$$
I_{M, \sigma}:=I_{M^{\prime}, \sigma^{\prime}}+I_{M^{\prime}, \sigma^{\prime \prime}} .
$$

If the surgery matrix of $M$ is diagonalizable, then (13) coincides with (12), since $I_{M, \sigma}$ is multiplicative with respect to the connected sum. Indeed, we have

$$
I_{M, \sigma}=I_{M, \sigma}\left(I_{\mathbb{R} P^{3}, \sigma_{0}}+I_{\mathbb{R} P^{3}, \sigma_{1}}\right)=I_{M, \sigma}\left(\frac{1}{1-v^{-1}}+\frac{-v^{-1}}{1-v^{-1}}\right) .
$$

Let $h \in H^{1}\left(M^{\prime}, \mathbb{Z} / 2 \mathbb{Z}\right)=\operatorname{Hom}\left(H_{1}\left(M^{\prime}, \mathbb{Z}\right), \mathbb{Z} / 2 \mathbb{Z}\right)$ be the class $v \mapsto$ $\nu(v, v)$, where $\nu$ is the linking pairing on $H_{1}\left(M^{\prime}, \mathbb{Z}\right)$. By Lemma 5.1, $\nu$ is a direct sum of $n+1$ copies of $\phi(2)$. Recall that $S_{k}=\left\{2^{k}(2 n+1) \mid n \in \mathbb{N}\right\}$. 
Set

$$
T=\bigcup_{k>2} S_{k} \quad \text { and } \quad T_{1}=\bigcup_{k>3} S_{k} .
$$

Proposition 6.1. For $M \in \mathcal{M}_{n}$ and a spin structure $\sigma$ on $M$, there exists a unique invariant $I_{M, \sigma}(v) \in \Gamma^{T}$ such that for any root of unity $\zeta$ with $\operatorname{ord}(\zeta) \in T_{1}, \operatorname{ev}_{\xi}\left(I_{M, \sigma}(v)\right)=\tau_{M, \sigma}^{\prime}(\xi)$. If $\operatorname{ord}(\zeta) \in S_{3}$, then $\operatorname{ev}_{\xi}\left(I_{M, \sigma}(v)\right)=$ $\tau_{M^{\prime}, \sigma^{\prime}+h}^{\prime}(\xi)+\tau_{M^{\prime}, \sigma^{\prime \prime}+h}^{\prime}(\xi)$.

Proposition 6.1 implies Theorem 4.

Proof. Let us first show that $I_{M, \sigma_{\varepsilon}}(v) \in \mathbb{Z}[1 / 2][v]^{T}$. Recall that $\left(\Phi_{n}\right)+$ $\left(\Phi_{m}\right)=(1)$ in $\mathbb{Z}[1 / 2][v]$ if and only if $n$ and $m$ are not adjacent in Habiro's sense. The denominator of (12) consists of all $\Phi_{i}(v)$ with $i \mid 4 j+2, j \in \mathbb{N}$. But such an $i$ is not adjacent to elements of $T$, i.e. $\Phi_{i}(v)$ is invertible in $\Gamma^{T}$ for all $i$.

We next show that the evaluation $I_{M, \sigma_{\varepsilon}}(v)$ coincides with the renormalized refined Witten-Reshetikhin-Turaev invariant. We again use the Laplace transform method.

For $k=3$, we define the refined Laplace transforms $L_{ \pm 2 ; \lambda}^{\varepsilon}: \mathbb{Z}\left[q^{ \pm \lambda}, q^{ \pm 1}\right] \rightarrow$ $\mathbb{Z}\left[q^{ \pm 1 / 2}\right]$ as the $\mathbb{Z}\left[q^{ \pm 1}\right]$-linear operators with

$$
\begin{aligned}
& L_{ \pm 2 ; \lambda}^{0}\left(q^{a \lambda}\right)= \begin{cases}v^{\mp a^{2}} & \text { for } a \text { odd } \\
0 & \text { otherwise }\end{cases} \\
& L_{ \pm 2 ; \lambda}^{1}\left(q^{a \lambda}\right)= \begin{cases}v^{\mp a^{2}} & \text { for } a \text { even } \\
0 & \text { otherwise }\end{cases}
\end{aligned}
$$

For $k>3$, the previous definitions of $L_{ \pm 2 ; \lambda}^{0}$ and $L_{ \pm 2 ; \lambda}^{1}$ should be interchanged. Then, for any Laurent polynomial $F\left(q^{\lambda}, q\right)$ and any root of unity $\zeta$ with $\operatorname{ord}(\zeta) \in S_{k}$, the following equation holds:

$$
\gamma_{ \pm 2}(\xi) \operatorname{ev}_{\xi}\left(L_{b ; \lambda}^{\varepsilon}\left(F\left(q^{\lambda}, q\right)\right)\right)=\sum_{\lambda}^{\xi, \varepsilon} q^{ \pm\left(\lambda^{2}-1\right) / 2} F\left(q^{\lambda}, q\right) .
$$

To simplify notation, we will sometimes drop the index $\lambda$ and denote $L_{ \pm 2 ; \lambda}^{\varepsilon}$ simply by $L_{ \pm 2}^{\varepsilon}$. Note that $\gamma_{ \pm 2}(\xi)=\gamma_{ \pm 2}^{1}(\xi)$ if $k=3$ and $\gamma_{ \pm 2}(\xi)=\gamma_{ \pm 2}^{0}(\xi)$ if $k>3$. In addition, we have

$$
\begin{aligned}
\left(L_{ \pm 2}^{1}-L_{ \pm 2}^{0}\right)\left(q^{a \lambda}\right) & =(-1)^{a}(-1)^{\chi+1} L_{ \pm 2}\left(q^{a \lambda}\right)=(-1)^{\chi+1}(-1)^{a} v^{\mp a^{2}} \\
& =\left.(-1)^{\chi+1} L_{ \pm 2}\right|_{v \rightarrow-v}\left(q^{a \lambda}\right)
\end{aligned}
$$

where $\chi=1$ if $k=3$ and zero otherwise. This allows us to express the refined Laplace transforms in terms of $L_{ \pm 2 ; \lambda}$ :

$$
L_{ \pm 2}^{\varepsilon}=\frac{1}{2}\left(L_{ \pm 2}+\left.(-1)^{\varepsilon+\chi} L_{ \pm 2}\right|_{v \rightarrow-v}\right) .
$$


Let us recall that $F_{L}^{0}(\xi)=F_{L}(\xi)$ (cf. [9], [3]).

The assertion in the diagonal case now follows from the next two formulas:

$$
\begin{aligned}
\operatorname{ev} \xi\left(\frac{\left.L_{2 ; \lambda}\left(F_{k}\right)\right|_{v \rightarrow-v}}{2\left(1-q^{-1}\right)}\right) \frac{\gamma_{2}(\xi)}{\gamma_{1}(\xi)} & =-\frac{\zeta^{-k}(1+\zeta)}{1-\zeta}\left(-\zeta^{2} ; \zeta\right)_{2 k} \tau_{\mathbb{R} P^{3}}(\xi), \\
\operatorname{ev}_{\xi}\left(\frac{\left.L_{-2 ; \lambda}\left(F_{k}\right)\right|_{v \rightarrow-v}}{2(1-q)}\right) \frac{\gamma_{-2}(\xi)}{\gamma_{-1}(\xi)} & =\frac{1+\zeta}{1-\zeta}\left(-\zeta^{2} ; \zeta\right)_{2 k} \tau_{\mathbb{R} P^{3}}(\xi) .
\end{aligned}
$$

Observe that $\tau_{M, \sigma}^{\prime}(\xi)=\tau_{M^{\prime}, \sigma^{\prime}}^{\prime}(\xi)+\tau_{M^{\prime}, \sigma^{\prime \prime}}^{\prime}(\xi)$. Uniqueness of $I_{M, \sigma}$ is provided by Proposition 2.2(a), (b).

Note that if $M \in \mathcal{M}_{1}$, then $I_{M, \sigma} \in \frac{1}{1-v} \widehat{\mathbb{Z}[v]_{s}}$. Thus we have the part of Theorem 6 concerning spin refinements.

6.2. Cohomological case. Let us first assume that $M \in \mathcal{M}_{n}$ can be obtained by surgery on an algebraically split link $L$ as above. Suppose $\sigma_{c} \in$ $H^{1}(M, \mathbb{Z} / 2 \mathbb{Z})$ is induced by the solution $c$ of the equation $L_{i j} c_{j}=0(\bmod 2)$.

We define

$$
\begin{aligned}
I_{M, \sigma_{c}}(v)= & \frac{(1+v)^{n}}{1-q} \sum_{k \geq 0}\left(q^{k+1}\right)_{k+1}\left(\sum_{\max k_{i}=k} C_{L, \mathbf{k}}(v)\right. \\
& \times \prod_{i=1}^{s_{+}}(-v)^{-k_{i}} B_{k_{i}}\left((-1)^{c_{i}+k_{i}+1} I, v\right) \\
& \left.\times \prod_{i=s_{+}+1}^{n} B_{k_{i}}\left((-1)^{c_{i}+1} I, v\right) \prod_{i=n+1}^{n+l_{+}}(-1)^{k_{i}} q^{-k_{i}\left(k_{i}+3\right) / 2}\right) .
\end{aligned}
$$

More generally, $M^{\prime}=M \# \mathbb{R} P^{3}$ can always be obtained by surgery on an algebraically split link. We set $I_{M, \sigma}:=I_{M^{\prime}, \sigma^{\prime}}+I_{M^{\prime}, \sigma^{\prime \prime}}$ where $\left.\sigma^{\prime}\right|_{M}=\left.\sigma^{\prime \prime}\right|_{M}=\sigma$.

TheOREM 8. For $M \in \mathcal{M}_{n}$ and a cohomology class $\sigma$ on $M$, there exists a unique invariant $I_{M, \sigma}(v) \in \Gamma^{S_{2}}$ such that for any $4 k$ th root of unity $\zeta$ with odd $k$ and $\zeta^{k^{2}}=(-1)^{\chi} I$, we have $\operatorname{ev}_{\xi}\left(I_{M, \sigma}(v)\right)=\tau_{M, \sigma+\chi h}^{\prime}(\xi)$.

Proof. We define the refined Laplace transforms as follows:

$$
L_{ \pm 2 ; \lambda}^{\varepsilon}\left(q^{a \lambda}\right)=\frac{1 \mp(-1)^{a+\varepsilon} \chi I}{2} v^{\mp a^{2}} .
$$

Then we have

$$
\gamma_{ \pm 2}(\xi) \operatorname{ev}_{\xi}\left(L_{ \pm 2 ; \lambda}^{\varepsilon}\left(q^{a \lambda}\right)\right)=\sum_{\lambda}^{\xi, \varepsilon} q^{ \pm\left(\lambda^{2}-1\right) / 2} q^{a \lambda} .
$$

By shifting $\lambda \mapsto \lambda+r / 2$, we see that $\gamma_{ \pm 2, r}^{1}= \pm \chi I \gamma_{ \pm 2, r}^{0}$. This allows us to 
express the refined Laplace transforms through the non-refined one:

$$
\begin{gathered}
L_{ \pm 2}^{1}\left(q^{a \lambda}\right)-L_{ \pm 2}^{0}\left(q^{a \lambda}\right)= \pm\left.\chi I L_{ \pm 2}\left(q^{a \lambda}\right)\right|_{v \rightarrow-v}, \\
L_{ \pm 2}^{\varepsilon}=\frac{1}{2}\left(L_{ \pm 2} \pm\left.(-1)^{\varepsilon+1} \chi I L_{ \pm 2}\right|_{v \rightarrow-v}\right) .
\end{gathered}
$$

The rest is analogous to the previous subsection.

\section{References}

[1] A. Beliakova, I. Bühler and T. Le, Unified $\mathrm{SO}(3)$ quantum invariants for rational homology 3-spheres, arXiv:0801.3893.

[2] A. Beliakova and T. Le, Integrality of quantum 3-manifold invariants and a rational surgery formula, Compos. Math. 143 (2007), 1593-1612.

[3] C. Blanchet, Invariants of three-manifolds with spin structure, Comm. Math. Helv. 67 (1992), 406-427.

[4] G. Gasper and M. Rahman, Basic Hypergeometric Series, Encyclopedia Math. Appl. 35, Cambridge Univ. Press, 1990.

[5] K. Habiro, A unified Witten-Reshetikhin-Turaev invariant for integral homology spheres, Invent. Math. 171 (2008), 1-81.

[6] - Cyclotomic completions of polynomial rings, Publ. RIMS 40 (2004), 1127-1146.

[7] - On the colored Jones polynomial of some simple links, in: Recent Progress Towards the Volume Conjecture (Kyoto, 2000), 34-43 (in Japanese).

[8] A. Kawauchi and S. Kojima, Algebraic classification of linking pairings on 3-manifolds, Math. Ann. 253 (1980), 29-42.

[9] R. Kirby and P. Melvin, The 3-manifold invariants of Witten and ReshetikhinTuraev for $\operatorname{sl}(2, \mathbb{C})$, Invent. Math. 105 (1991), 473-545.

[10] T. Le, Strong integrality of quantum invariants of 3-manifolds, Trans. Amer. Math. Soc. 360 (2008), 2941-2963.

[11] —, Quantum invariants of 3-manifolds: integrality, splitting, and perturbative expansion, in: Proc. Pacific Institute for the Mathematical Sciences Workshop "Invariants of Three-Manifolds" (Calgary, AB 1999), Topology Appl. 127 (2003), 125-152.

[12] - On perturbative PSU $(N)$ invariants of rational homology 3-spheres, Topology 39 (2000), 813-849.

[13] T. Le, J. Murakami and T. Ohtsuki, On a universal perturbative invariant of 3manifolds, ibid. 37 (1998), 539-574.

[14] G. Masbaum, Skein-theoretical derivation of some formulas of Habiro, Algebr. Geom. Topology 3 (2003), 537-556.

[15] G. Masbaum and J. Roberts, A simple proof of integrality of quantum invariants at prime roots of unity, Math. Proc. Cambridge Philos. Soc. 121 (1997), 443-454.

[16] H. Murakami, Quantum SU(2)-invariants of three-manifolds associated with the trivial first cohomology class modulo two, in: Low Dimensional Topology (Funchal, 1998), H. Nencka (ed.), Contemp. Math. 233, Amer. Math. Soc., 1999, 117-136; http://www3.tky.3web.ne.jp/\%7Estarshea/paper/madeira.ps.zip.

[17] -, Quantum SU(2)-invariants for three-manifolds associated with non-trivial cohomology classes modulo two, in: Knots in Hellas '98, Ser. Knots Everything 24, World Sci., 2000, 347-352; http://www3.tky.3web.ne.jp/\%7Estarshea/paper/hellas.ps.zip.

[18] T. Ohtsuki, A polynomial invariant of rational homology 3-spheres, Invent. Math. 123 (1996), 241-257. 
[19] V. Turaev, Quantum Invariants of Knots and 3-Manifolds, de Gruyter Stud. Math. 18, de Gruyter, 1994.

[20] C. Wall, Quadratic forms on finite groups, and related topics, Topology 2 (1963), 281-298.

Institut für Mathematik

Universität Zürich

Winterthurerstrasse 190

CH-8057 Zürich, Switzerland

E-mail: anna@math.unizh.ch

School of Mathematics

Georgia Institute of Technology

Atlanta, GA 30332-0160, U.S.A.

E-mail: letu@math.gatech.edu
Institut de Mathématiques de Jussieu

(UMR-CNRS 7586)

Université Paris Diderot 175 rue du Chevaleret F-75013 Paris, France E-mail: blanchet@math.jussieu.fr

Received 17 February 2007;

in revised form 12 June 2008 